\title{
Avaliação dos Desvios na Trajetória Originados pelo Acoplamento entre o Controle de Atitude e de Órbita em Manobras Orbitais com Propulsão Contínua
}

\author{
Evandro Marconi Rocco, Aguinaldo Cardozo da Costa Filho \\ Instituto Nacional de Pesquisas Espaciais, INPE \\ 12227-010, São José dos Campos, SP \\ E-mail: evandro.rocco@inpe.br, aguinaldoccf@gmail.com
}

\begin{abstract}
Resumo: Existem basicamente dois tipos de manobras orbitais: as que proporcionam a correção de órbita e as de transferência de órbita. Essas manobras exigem um sistema de controle que deverá ser constituído, dentre outras coisas, por subsistemas que atuem na órbita $e$ atitude. $O$ controle de atitude e órbita são subsistemas que podem interagir. Todavia, considerando um caso ideal, pode-se afirmar que não existe um acoplamento matemático entre atitude e órbita. Porém, na prática, o acoplamento ocorre quando o controle de órbita depende do apontamento de um propulsor fixo no veículo, que por sua vez depende da atitude. Assim, o controle de atitude deve seguir uma direção de referência fornecida pelo subsistema de controle de órbita. Mas os erros no controle de atitude, ou até mesmo o tempo de resposta do sistema, afetarão o controle de órbita. Portanto, o estudo da interação entre os sistemas de controle de atitude e de órbita faz-se necessário.
\end{abstract}

\section{Introdução}

Esse trabalho considera o problema do acoplamento entre o controle de trajetória e controle de atitude em manobras de transferência orbital utilizando um sistema propulsivo capaz de aplicar empuxo contínuo por um longo período de tempo. Os estados de atitude e órbita são propagados utilizando as equações da dinâmica do movimento. São analisados efeitos no sistema de controle que surgem durante a transferência orbital. É considerado um sistema de controle em malha fechada e propulsão de baixo empuxo com alto impulso específico. Não se está buscando neste trabalho especificar as causas das falhas e/ou perturbações, bem como especificar qual tipo de propulsão contínua deve ser utilizada. Com auxílio de simulações foi possível analisar o desvio na trajetória e avaliar o sistema de controle. O sistema precisa ser modular para poder permitir testes das estratégias de controle de forma separadas e posteriormente acopladas adequadamente.

Para manobras de correção e transferência de órbita, normalmente utiliza-se um controle em malha aberta comandado por terra. Entretanto, em algumas missões, como por exemplo do tipo drag-free, Rocco (2012), o controle orbital em malha fechada é necessário. Estas missões geralmente utilizam micro-propulsores como atuadores para o subsistema de controle de órbita. O sistema de controle deverá ser constituído, dentre outras coisas, por subsistemas que atuem na órbita e na atitude. Entretanto, considerando um caso ideal, pode-se afirmar que não existe um acoplamento matemático entre atitude e órbita. O controle de atitude e da trajetória de um veículo espacial são problemas que comumente são tratados de formas separadas. Em particular, assume-se frequentemente que a dinâmica da órbita e a dinâmica da atitude são independentes. Porém, o acoplamento ocorre quando o controle da órbita depende do apontamento de um propulsor fixo no veículo, que por sua vez depende da atitude. Assim, o controle de atitude deve seguir uma direção de referência fornecida pelo subsistema de controle de órbita. Mas os erros no controle de atitude, ou até mesmo o tempo de resposta do sistema, afetarão o controle de órbita. Por outro lado, o controle orbital pode interferir na atitude do veículo se durante uma manobra surgirem torques devido a erros na direção do empuxo de forma a aplicá-lo fora do centro de massa do veículo. Portanto, o estudo da interação entre os sistemas de controle de atitude e de órbita faz-se necessário. Chodas (1982) afirma que é particularmente apropriado combinar a órbita e a determinação da atitude. A abordagem 
proposta por Chodas (1982) considera o acoplamento órbita/atitude por um modelo explícito que contempla torques externos e perturbações de forças perturbadoras. Neste trabalho utilizase abordagem semelhante com o objetivo de estudar o acoplamento, ou interação, entre o controle de atitude e de trajetória durante manobras orbitais de empuxo contínuo para satélites artificiais, utilizando para isto o ambiente de simulação Spacecraft Trajectory and Atitude Simulator (STAS) (Rocco, 2008, Costa Filho, 2010 e Rocco et al. 2011).

\section{Acoplamento entre o controle de atitude e de órbita}

De acordo com Lennox (2004), o acoplamento dos sistemas de controle de órbita e atitude é um conceito relativamente novo e existem poucas publicações disponíveis. A negligencia deste acoplamento, no que se refere ao controle de órbita, usualmente é justificada pelo fato de que a simulação da dinâmica de atitude utiliza uma taxa de amostragem maior do que a simulação da dinâmica orbital. Dessa forma, a atitude poderia ser ajustada adequadamente antes do acionamento dos propulsores de manobra orbital de maneira que não houvessem erros no apontamento dos propulsores. Entretanto nem sempre isso é possível. Existem atrasos e não linearidades, que são características inerentes de todos os sistemas de controle, que podem impedir o correto apontamento dos propulsores devido ao erro no controle de atitude.

Neste trabalho considerou-se o controle em malha fechada tanto para o controle da atitude quanto para o controle da trajetória, Figuras 1,2 e 3.

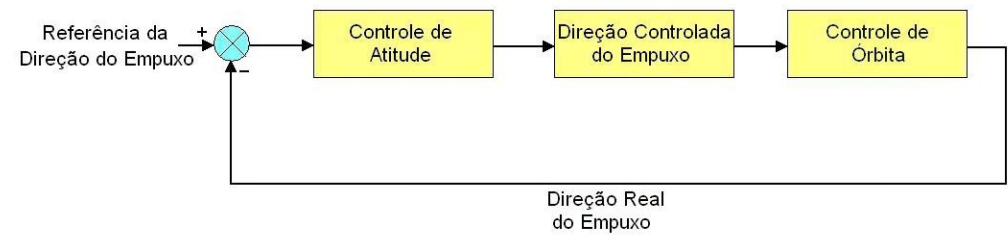

Figura 1: Controle de atitude e órbita.

O acoplamento entre os subsistemas de controle de atitude e órbita ocorre por meio dos requisitos de apontamento do propulsor, definidos pela dinâmica do movimento orbital que, por sua vez, define a atitude necessária para que o empuxo seja aplicado corretamente de maneira a controlar a trajetória do veículo. Portanto o erro de apontamento do propulsor é função do erro gerado pelo controle de atitude, e assim, o controle orbital depende diretamente do controle de atitude, como ilustrado nas Figuras 2 e 3.

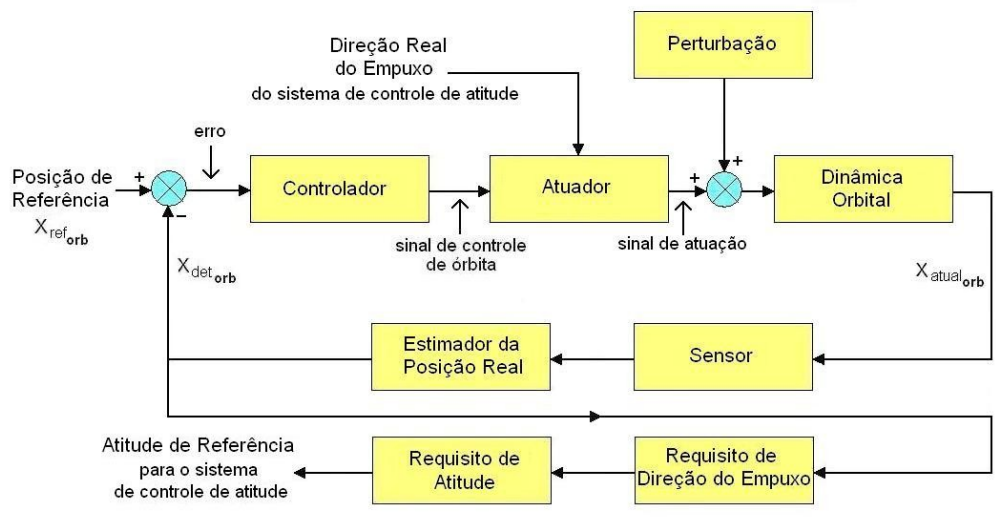

Figura 2: Subsistema de controle de órbita.

O subsistema de controle de órbita considera a direção real de apontamento do propulsor de acordo com a atitude do veículo. Com essa informação a direção do empuxo necessária para a manobra orbital é calculada e os requisitos de apontamento são determinados, esses requisitos são então fornecidos ao controle de atitude e passam a ser considerados como a atitude de referência a ser perceguida pelo subsistema de controle de atitude. Em seguida, a direcção de 
impulso efetiva é fornecida para o subsistema de controle de órbita fechando o ciclo de controle.

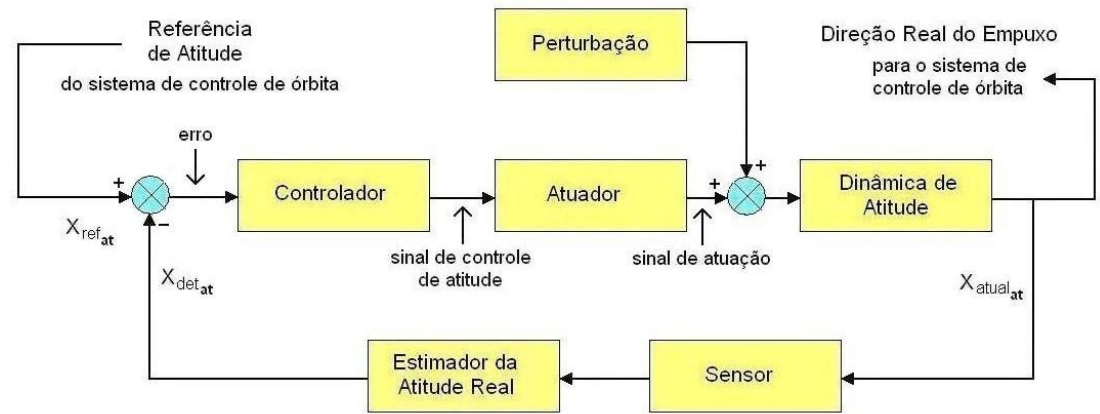

Figura 3: Subsistema de controle de atitude.

\section{Referencial adotado}

Para a órbita, o vetor de estado (posição e velocidade) é calculado considerando-se o referencial inercial geocêntrico (ECI) referente ao equinócio vernal J2000. Para a atitude do veículo espacial são utilizados os ângulos de Euler que definem as rotações em torno dos eixos de rolamento (roll), arfagem ( pitch) e guinada (yaw). Adotou-se o eixo de guinada voltado para o nadir, o eixo de arfagem na direção normal ao plano da órbita e o eixo de rolamento perpendicular aos outros dois, conforme a Figura 4.

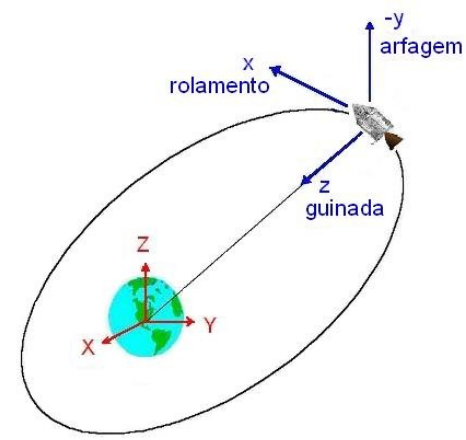

Figura 4: Referencial.

\section{Modelagem matemática}

\section{1 Órbita}

O movimento orbital pode ser simulado por meio da solução da equação de Kepler para cada passo da simulação. Portanto, dado um estado inicial e um intervalo de tempo (passo de simulação), o estado pode ser convertido para elementos keplerianos, e usando a equação de Kepler, os elementos são propagados, considerando o intervalo de tempo dado. Em seguida, os novos elementos keplerianos podem ser convertidos para o novo estado. $\mathrm{O}$ processo repete-se para o passo seguinte propagando a trajetória ao longo do tempo. Como mostram as Equações 1 a 12 (Bate et al.,1971) em que $\mu$ é a constante gravitacional, $a$ é o semi-eixo maior, $e$ é a excentricidade da órbita, $\Omega$ é a ascensão reta do nodo ascendente, $i$ é a inclinação, $\omega$ é o argumento do perigeu e $f$ é a anomalia verdadeira.

$$
\begin{aligned}
& M=u-e \sin u=\sqrt{\frac{\mu}{a^{3}}}(t-T) \\
& r=\left(X^{2}+Y^{2}+Z^{2}\right)^{1 / 2} ; \quad r=a(1-e \cos u) \\
& v=\left(\dot{X}^{2}+\dot{Y}^{2}+\dot{Z}^{2}\right)^{1 / 2} ; \quad v^{2}=\mu\left(\frac{2}{r}-\frac{1}{a}\right)
\end{aligned}
$$




$$
\begin{aligned}
& X_{i}=A_{i}(\cos u-e)+B_{i} \sin u ; \quad \dot{X}_{i}=\frac{a_{n}}{r}\left(-A_{i} \sin u+B_{i} \cos u\right) \\
& A_{x}=a R_{11} ; B_{x}=a \sqrt{1-e^{2}} R_{12} ; A_{y}=a R_{21} ; B_{y}=a \sqrt{1-e^{2}} R_{22} ; A_{z}=a R_{31} ; B_{z}=a \sqrt{1-e^{2}} R_{32} \\
& \boldsymbol{R}(\Omega, i, \omega)=\left[\begin{array}{lll}
R_{11} & R_{12} & R_{13} \\
R_{21} & R_{22} & R_{23} \\
R_{31} & R_{32} & R_{33}
\end{array}\right] ;\left\{\begin{array}{l}
R_{11}=\cos \omega \cos \Omega-\sin \omega \sin \Omega \cos i \\
R_{12}=-\sin \omega \cos \Omega-\cos \omega \sin \Omega \cos i \\
R_{13}=\sin \Omega \sin i \\
R_{21}=\cos \omega \sin \Omega+\sin \omega \cos \Omega \cos i \\
R_{22}=-\sin \omega \sin \Omega+\cos \omega \cos \Omega \cos i \\
R_{23}=-\sin i \cos \Omega \\
R_{31}=\sin \omega \sin i \\
R_{32}=\cos \omega \sin i \\
R_{33}=\cos i
\end{array}\right. \\
& \vec{h}=\vec{r} \times \vec{v}=(Y \dot{Z}-\dot{Y} Z) \hat{i}+(Z \dot{X}-\dot{Z} X) \hat{j}+(X \dot{Y}-\dot{X} Y) \hat{k} ; \quad r \dot{r}=X \dot{X}+Y \dot{Y}+Z \dot{Z} \\
& e=\left[\left(\frac{r \dot{r}}{n a^{2}}\right)^{2}+\left(1-\frac{r}{a}\right)^{2}\right]^{1 / 2} ; \quad i=\arctan \left[\frac{\left(h_{X}^{2}+h_{Y}^{2}\right)^{1 / 2}}{h_{Z}}\right] \\
& \Omega=\arctan \left(\frac{h_{X}}{-h_{Y}}\right) ; \quad f=\arcsin \left(\frac{\left(1-e^{2}\right)^{1 / 2} \sin u}{1-e \cos u}\right)=\arccos \left(\frac{\cos u-e}{1-e \cos u}\right) \\
& \tan \theta=\frac{-\cos i \sin \Omega X+\cos i \cos \Omega Y+\sin i Z}{\cos \Omega X+\sin \Omega Y} ; \quad \theta=\omega+f
\end{aligned}
$$

\subsection{Atitude}

Considerando-se o veículo como um corpo rígido, o movimento de atitude pode ser simulado de maneira simplificada usando as equações de Euler. Neste estudo, foram adotados os eixos x, y e z de maneira a coincidir com os eixos principais de inércia. Dados os momentos de inércia principais, as velocidades angulares iniciais e os torques aplicados, as acelerações angulares são obtidas. Com as posições iniciais (ângulos de Euler) e o passo da simulação as novas posições angulares são calculadas. Como mostram as Equações 13 a 16 (Hughes, 2004), em que $\bar{I}_{x}, \bar{I}_{y}$ e $\bar{I}_{z}$ são os momentos de inércia, $M_{x}, M_{y}$ e $M_{z}$ são os torques aplicados, $\dot{\omega}_{x}$, $\dot{\omega}_{y}$ e $\dot{\omega}_{z}$ as acelerações angulares, $\omega_{x}, \omega_{y}$ e $\omega_{z}$ as velocidades angulares e $\alpha_{x}, \alpha_{y}$ e $\alpha_{z}$ os ângulos de Euler (ângulos de rolamento, arfagem e guinada).

$$
\begin{aligned}
& \sum M_{x}=\bar{I}_{x} \dot{\omega}_{x}-\left(\bar{I}_{y}-\bar{I}_{z}\right) \omega_{y} \omega_{z} \\
& \sum M_{y}=\bar{I}_{y} \dot{\omega}_{y}-\left(\bar{I}_{z}-\bar{I}_{x}\right) \omega_{z} \omega_{x} \\
& \sum M_{z}=\bar{I}_{z} \dot{\omega}_{z}+\left(\bar{I}_{x}-\bar{I}_{y}\right) \omega_{x} \omega_{y} \\
& \dot{\omega}_{x}=\frac{M_{x}+\left(\bar{I}_{y}-\bar{I}_{z}\right) \omega_{y 0} \omega_{z 0}}{\bar{I}_{x}} ; \dot{\omega}_{y}=\frac{M_{y}+\left(\bar{I}_{z}-\bar{I}_{x}\right) \omega_{y 0} \omega_{x 0}}{\bar{I}_{y}} ; \dot{\omega}_{z}=\frac{M_{z}+\left(\bar{I}_{x}-\bar{I}_{y}\right) \omega_{x 0} \omega_{y 0}}{\bar{I}_{z}} \\
& \alpha_{x}=\alpha_{x 0}+\omega_{x} t+\frac{1}{2} \dot{\omega}_{x} t^{2} ; \alpha_{y}=\alpha_{y 0}+\omega_{y} t+\frac{1}{2} \dot{\omega}_{y} t^{2} ; \alpha_{z}=\alpha_{z 0}+\omega_{z} t+\frac{1}{2} \dot{\omega}_{z} t^{2} \\
& \omega_{x}=\omega_{x 0}+\dot{\omega}_{x} t ; \omega_{y}=\omega_{y 0}+\dot{\omega}_{y} t ; \omega_{z}=\omega_{z 0}+\dot{\omega}_{z} t
\end{aligned}
$$




\section{Simulação e resultados}

A simulação tem como objetivo avaliar a dinâmica do movimento orbital levando em conta a dinâmica de atitude para o apontamento do vetor de empuxo. Considerou-se que o empuxo utilizado na manobra orbital foi aplicado na direção tangencial à trajetória, a fim de alterar o semi-eixo maior da órbita. Para testar o subsistema de controle de órbita foram considerados perturbações exageradas (pequenos pulsos) e erros aleatórios de variância 0,00001 e viés de $0,005 \mathrm{~m} / \mathrm{s}$ para os propulsores e variância 0,0001 e viés de $0,0001 \mathrm{~m} / \mathrm{s}$ para os sensores. Os elementos keplerianos da órbita inicial são dados por: $a=9000 \mathrm{~km}, e=0,01, \Omega=15^{\circ}$, $i=45^{\circ}, \omega=5^{\circ}$ e anomalia média $M=0$. Partindo da órbita inicial o empuxo aplicado foi de $2 \mathrm{~N}$ durante o período simulado de $10800 \mathrm{~s}$. Os pulsos perturbadores foram aplicados, nos três eixos, nos instantes $250 \mathrm{~s}, 500 \mathrm{~s}$ e $750 \mathrm{~s}$.

Devido aos pulsos perturbadores o subsistema de controle de atitude deve reagir girando o veículo de maneira a ajustar o apontamento do vetor de empuxo para que os erros inseridos pela perturbação sejam mitigados. Entretanto, para testar o subsistema de controle de atitude, também foram consideradas incertezas nos sensores e atuadores deste subsistema: erros aleatórios de variância 0,1 e viés de $0,05 \mathrm{~m} / \mathrm{s}$ mais sinal senoidal de amplitude $0,0001 \mathrm{~m} / \mathrm{s}$ e frequência $0,0001 \mathrm{rad} / \mathrm{s}$ para os sensores; e para os atuadores além da incerteza devido ao erro aleatório de variância 0,00001 e viés de $0,05 \mathrm{~m} / \mathrm{s}$ considerou-se uma zona morta de $+/-0,005^{\circ} \mathrm{e}$ um limite máximo para a taxa de variação do sinal de atuação de $20 \%$ s.

Portanto, durante a manobra orbital a atitude deve ser controlada para manter a orientação correta dos propulsores. Assim, a atitude deve mudar ao longo da órbita. Os resultados da simulação são apresentados nas Figuras 5 a 11.

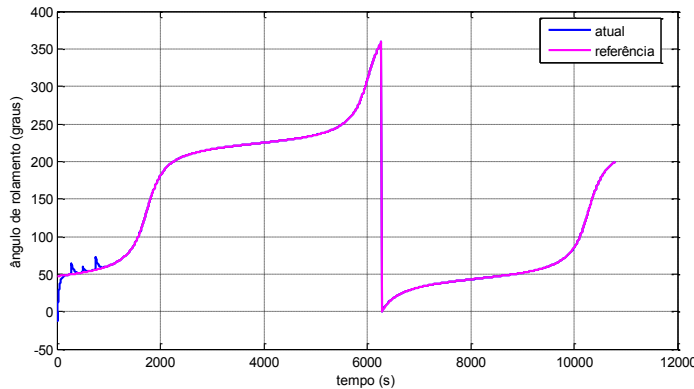

Figura 5: Posição angular atual e de referência para o eixo de rolamento (graus).

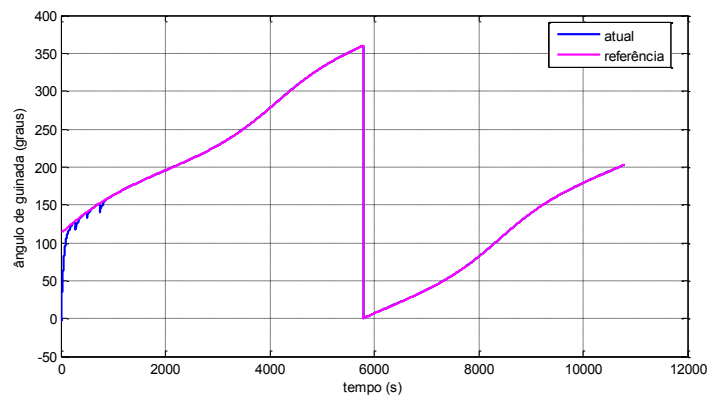

Figura 7: Posição angular atual e de referência para o eixo de guinada (graus).

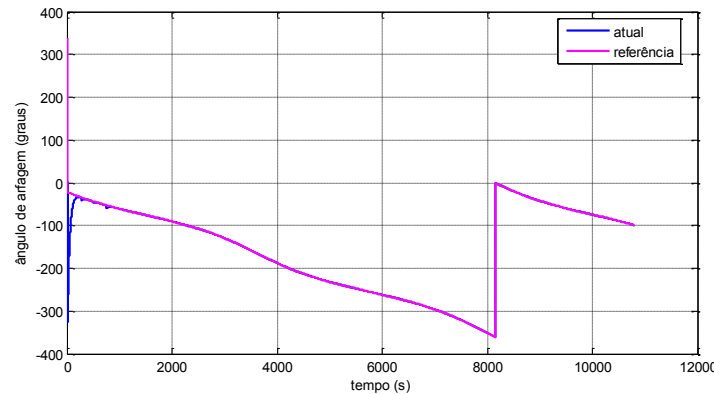

Figura 6: Posição angular atual e de referência para o eixo de arfagem (graus).

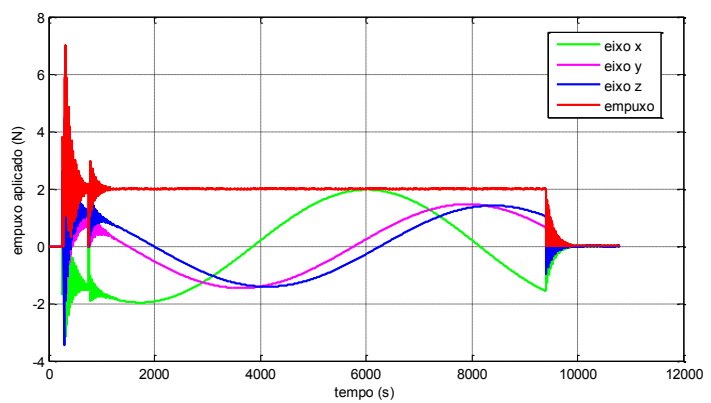

Figura 8: Empuxo aplicado durante a manobra orbital $(\mathrm{N})$.

Durante a manobra de órbita, os desvios na velocidade orbital ocorrem devido aos pulsos perturbadoras e aos erros nos sensores e atuadores dos subsistemas de órbita e atitude. A Figura 8 ilustra esse desvio onde nota-se o regime transitório gerado pela aplicação dos pulsos perturbadores. No entanto, com a atuação do sistema de controle o desvio tende a zero ao final do transitório demonstrando a eficácia do sistema de controle. 


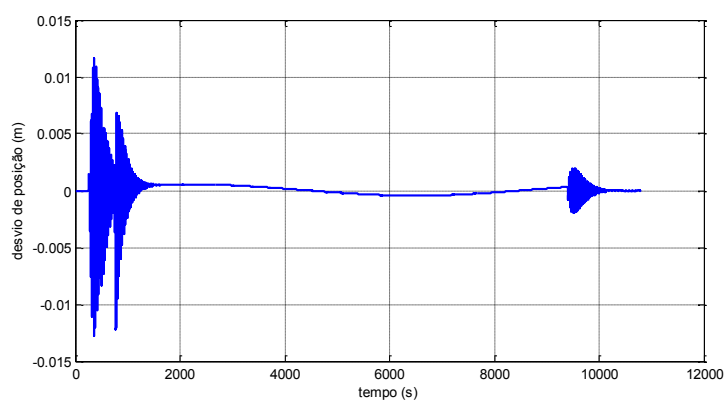

Figura 9: Desvio na posição orbital (m).

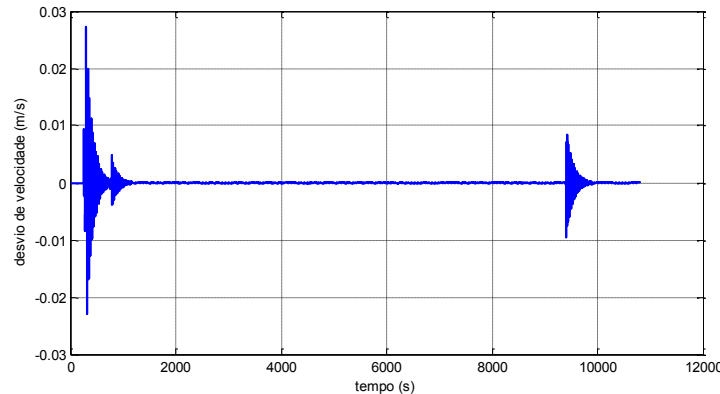

Figura 10: Desvio na velocidade orbital $(\mathrm{m} / \mathrm{s})$.

Devido aos erros na atitude, ocorrem desvios nos elementos keplerianos, que são mostrados na Figura 11. No entanto ao final da manobra, mesmo considerando esses desvios na atitude e os erros nos sensores e atuadores de ambos os subsistema, os desvios na órbita foram reduzidos para níveis aceitáveis.
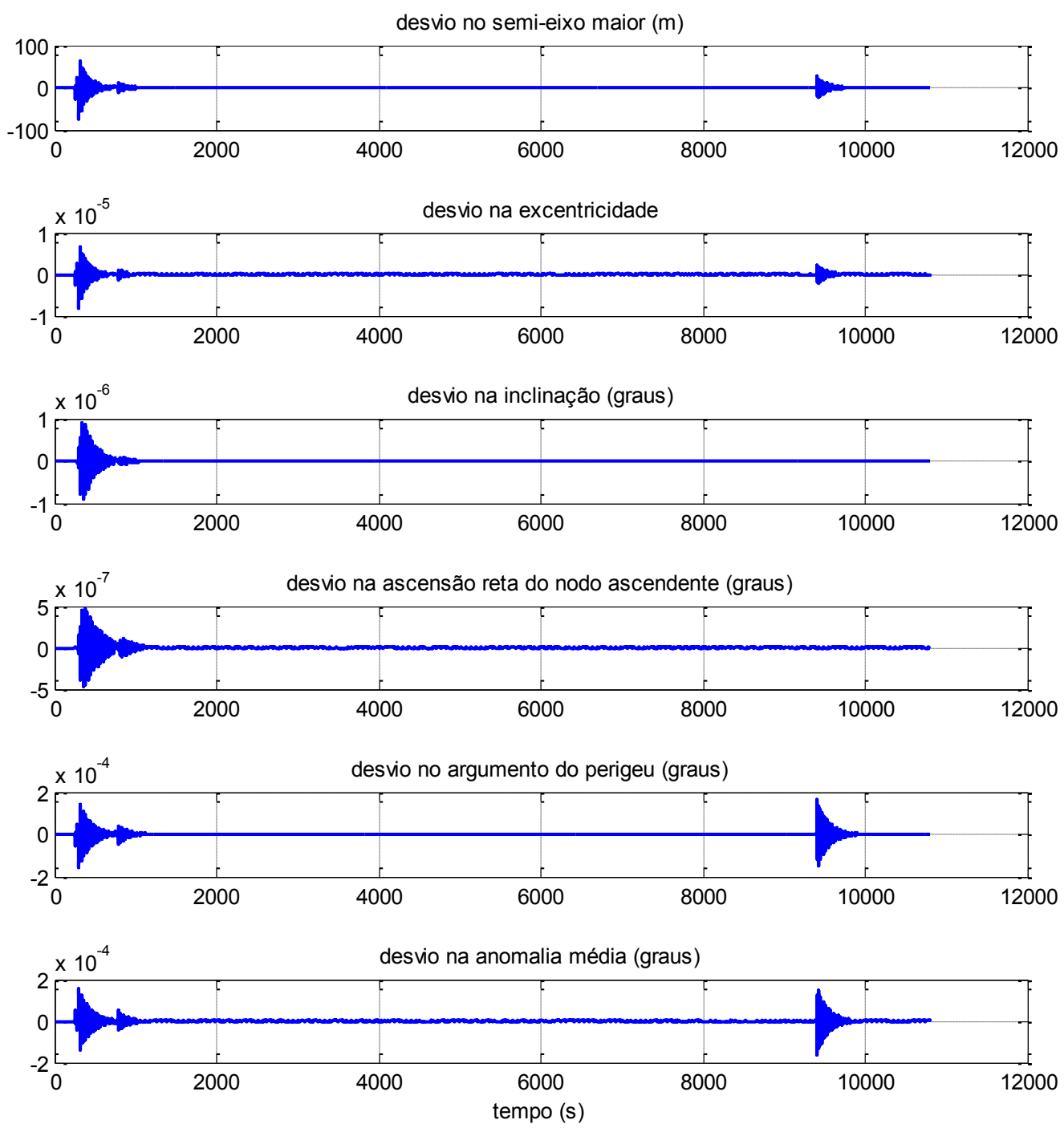

Figura 11: Desvio nos elementos keplerianos. 


\section{Conclusão}

Um modelo capaz de considerar o efeito dos erros na atitude e na trajetória durante manobras orbitais foi implementado e testado com sucesso. Os resultados mostram que o modelo é capaz de determinar os desvios nos elementos keplerianos e no estado de um veículo espacial sujeito a erros no controle de atitude. O erro na trajetória devido aos erros na atitude deve ser considerado na análise de missão de um veículo espacial. O desvio na atitude provoca um desvio na direção do empuxo aplicado. Portanto, a trajetória deve ser corrigida continuamente, o que provoca um aumento do consumo de combustível. Em uma missão real é de fundamental importância avaliar o gasto de combustível e/ou energia para mitigar todos os possíveis desvios tanto na trajetória quanto na atitude. Assim, este trabalho apresenta uma ferramenta que pode ser útil na análise e desenvolvimento de futuras missões espaciais.

\section{Referências}

[1] Rocco, E. M. Controle de trajetória com propulsão contínua para missões do tipo drag-free. In: Congresso Nacional de Engenharia Mecânica, 7, 2012, São Luís, Brasil, 2012.

[2] Chondas, P. W. Combined satellite attitude and orbit determination with dynamic coupling. In: American Institute of Aeronautics and Astronautics and American Astronautical society. Astrodynamics Conference. San Diego, CA, Aug. 9-11, 1982. AIAA Paper 82-1419.

[3] Rocco, E. M. Perturbed orbital motion with a PID control system for the trajectory. In: Colóquio Brasileiro de Dinâmica Orbital, 14, Águas de Lindóia, 2008. Resumos. 2008b

[4] Costa Filho, A.C. Análise de Acoplamento entre o Controle de Atitude e de Trajetória durante Manobras Orbitais de Empuxo Contínuo, INPE, São José dos Campos, 113p. Dissertação de mestrado, Instituto Nacional de Pesquisas Espaciais, 2010.

[5] Rocco, E. M.; Costa Filho, A.C; Carrara, V., 2011, Effect the coupling between attitude and orbital control in maneuvers using continuous thrust. Minissimpósio: Aerospace Engineering. In: $10^{\text {a }}$. Conferência Brasileira de Dinâmica, Controle e Aplicações.

[6] Lennox, S. E., Coupled Attitude and Orbital Control System Using Spacecraft Simulators. Master Thesis in Aerospace Engineering, Virnia Polytechnic Institute and State University, Virginia-USA, 2004.

[7] Bate, R. R.; Mueller, D. D.; White, J. E. Fundamentals of Astrodynamics. Dover Publications, Inc., New York, 1971.

[8] Hughes, P. C. Spacecraft Attitude Dynamics. Dover Publications, Inc., New York, 2004. 